\title{
Reconexiones en dispositivos conexiales: La reconstrucción de lo religioso en redes socio- digitales
}

\section{Reconnections in connectional devices: The reconstruction of the religious in socio-digital networks}

\author{
Moisés Sbardelotto ${ }^{1}$ \\ Universidade do Vale do Rio dos Sinos (Unisinos), Brasil \\ msbardelotto@yahoo.com.br
}

Recibido: 10 de septiembre 2013

Aceptado y Publicado: 24 de noviembre de 2013

\begin{abstract}
Resumen
En las interacciones sociales tecnológicamente mediadas del ambiente digital, las prácticas sociales revelan dinámicas mediatizadas, que también involucran a las operaciones de las instituciones sociales, como la Iglesia, y sus miembros. En esos ambientes, hay numerosos sentidos religiosos en circulación. Ocurre, de esa forma, un desvío en la práctica de la fe al ambiente online, a partir de lógicas mediáticas, complejificando el fenómeno religioso y las procesualidades comunicacionales. Se forman así nuevas modalidades de percepción y de expresión del sagrado en nuevos ambientes de culto. No solamente las instituciones eclesiales o las instituciones mediáticas, sino también la sociedad en general, en los más diversos ámbitos de Internet, hablan sobre lo religioso. En este artículo, presentamos una reflexión sobre la mediatización digital de la religión, fenómeno social contemporáneo en que se sitúa la reconstrucción de lo religioso. Analizamos los conceptos de reconexión y dispositivos conexiales, a partir de las interacciones entre usuarios de páginas de Facebook que abordan constructos católicos. Como conclusión, apuntamos que, en esa reconstrucción de 10 "católico", surge una religiosidad en experimentación marcada por e-rejías, o sea, nuevos sentidos simbólicos de lo religioso en red, "bricolajes de la fe" en el ambiente digital.
\end{abstract}

\footnotetext{
${ }^{1}$ Máster y doctorando en Ciencias de la Comunicación en la Universidade do Vale do Rio dos Sinos (Unisinos), en la línea de investigación Mediatización y Procesos Sociales. Becario del Conselho Nacional de Desenvolvimento Científico e Tecnológico de Brasil (CNPq). Es graduado en Comunicación Social - Periodismo en la Universidade Federal do Rio Grande do Sul (UFRGS). Correo electrónico: msbardelotto@yahoo.com.br.
} 


\section{Summary}

In the technologically mediated social interactions of the digital environment, social practices reveal mediated dynamics, which also involve the operations of social institutions, such as the Church, and its members. In these environments, there are numerous religious meanings in circulation. In this way, there is a deviation in the practice of faith to the online environment, based on media logics, making the religious phenomenon and communicational processes more complex. Thus, new modes of perception and expression of the sacred are formed in new cult environments. Not only ecclesial institutions or media institutions, but also society in general, in the most diverse areas of the Internet, speak about religion. In this article, we present a reflection on the digital mediatization of religion, a contemporary social phenomenon in which the reconstruction of religion is situated. We analyze the concepts of reconnection and connectional devices, based on the interactions between users of Facebook pages that address Catholic constructs. In conclusion, we point out that, in this reconstruction of the "catholic", a religiosity in experimentation arises marked by e-rejías, that is, new symbolic meanings of the religious in network, "bricolajes de la fe" in the digital environment.

Palabras claves: Dispositivos conexiales, Redes sociales, Interacciones sociales, Tecnología, fenómeno religioso.

Keywords: Connectional devices, Social networks, Social interactions, Technology, religious phenomenon.

\section{Introducción}

En las redes socio-digitales, ambientes online de sociabilidad, se manifiestan "puntos" de interacción y de intensos intercambios comunicacionales, atemporales e aespaciales, entre los internautas. En esas interacciones tecnológicamente mediadas, las prácticas sociales revelan lógicas mediatizadas, que también involucran a las operaciones de las instituciones sociales, como la Iglesia, y sus miembros. Ocurre así un desvío en la práctica de la fe al ambiente online, a partir de lógicas mediáticas, complejificando el fenómeno religioso y las procesualidades comunicacionales. Se forman así nuevas modalidades de percepción y de expresión del sagrado en nuevos ambientes de culto, caracterizando un fenómeno de mediatización digital de la religión (Sbardelotto, 2012).

En esos ambientes, hay numerosos sentidos religiosos en circulación. Esa circulación, todavía, no es una acción restricta de las instituciones eclesiales, o de las instituciones mediáticas; sino

Facultad de Ciencias de la Información - Universidad de La Laguna

Avenida César Manrique, s/n; Campus de Guajara

38071 La Laguna, Tenerife (Islas Canarias - España) 
también la sociedad en general, en los más diversos ámbitos de Internet, habla públicamente sobre lo "religioso" - en nuestro caso específico de interés, sobre lo "católico", es decir, constructos simbólicos que la sociedad en su conjunto considera como vinculados a las creencias y a las prácticas de la Iglesia Católica. Lo "sagrado" circula y fluye a través de la complejidad de internet por medio de una acción no sólo del ámbito de la "producción" eclesial, sino también mediante una acción comunicacional de los numerosos usuarios en ambientes fluidos "entre lo privado y lo público; entre la institución y el individuo; entre la autoridad y la autonomía individual; entre los grandes marcos mediáticos y el prosumo [prosumption] individual" (Hoover \& Echchaibi, 2012, p.16, traducción nuestra).

Así se resignifican socialmente la experiencia, la doctrina y la tradición católicas vía medios digitales. Los individuos "re-construyen" lo que fue construido por la institución (eclesial o mediática), poniéndolo en circulación, y también construyen ellos mismos sentidos relativos a lo sagrado en general o a la institución que a su vez los "re-construye", y así sucesivamente. Ahí podemos percibir la circulación como el "dispositivo central" del proceso de comunicación (Fausto Neto, 2010).

En este artículo, presentamos una reflexión sobre la mediatización digital de la religión, fenómeno social contemporáneo en que se sitúa la reconstrucción de lo religioso. Proponemos aún un análisis de los conceptos de reconexión y dispositivos conexiales, a partir de las interacciones entre usuarios de páginas de Facebook vinculadas a la experiencia, la doctrina y la tradición católicas. Como conclusión, vemos que, en esa reconstrucción social de lo "católico", surge una religiosidad en experimentación marcada por e-rejías, o sea, nuevos sentidos simbólicos de lo religioso en red, "bricolajes de la fe" en el ambiente digital.

\section{Mediatización digital de la religión: fundamentos}

La Iglesia Católica, aún en 2012, en un encuentro mundial de obispos, abordó de manera central el tema de la "nueva evangelización" - o sea, de su presencia y anuncio en las sociedades contemporáneas. En el documento final, se habla de los medios de comunicación, incluso digitales, reconociendo su rol en la "transformación de la misma realidad".

Las nuevas tecnologías digitales han dado origen a un verdadero y nuevo espacio social, cuyas relaciones son capaces de influenciar sobre la sociedad y sobre la cultura. Al ejercer una influencia sobre la vida de las personas, los procesos mediáticos, que son factibles con estas tecnologías, Ilegan a transformar la misma realidad. Intervienen 
en modo incisivo en la experiencia de las personas y permiten una dilatación de las potencialidades humanas. La percepción de nosotros mismos, de los otros y del mundo dependen del influjo que tales tecnologías ejercen. (Sínodo, 2012, s/p, subrayados nuestros).

Así, la propia Iglesia reconoce que los procesos socio-técnicos impulsados por las nuevas tecnologías digitales favorecen el surgimiento de una nueva sociedad y de una nueva cultura. La realidad y la experiencia de la realidad son transformadas. Algo semejante fue reconocido por el papa emérito, Benedicto XVI, en uno de sus últimos documentos oficiales, el Mensaje para la 47a Jornada Mundial de las Comunicaciones Sociales, intitulada "Redes Sociales: portales de verdad y de fe; nuevos espacios para la evangelización".

Quisiera detenerme a considerar el desarrollo de las redes sociales digitales, que están contribuyendo a que surja una nueva «ágora», una plaza pública y abierta en la que las personas comparten ideas, informaciones, opiniones, y donde, además, nacen nuevas relaciones y formas de comunidad. [...] Las redes se convierten así, cada vez más, en parte del tejido de la sociedad [...].Las redes sociales son el fruto de la interacción humana pero, a su vez, dan nueva forma a las dinámicas de la comunicación que crea relaciones (Benedicto XVI, 2012, s/p, subrayado nuestro).

Como vemos, el papa emérito también percibe que las nuevas prácticas sociales que surgen con la apropiación de las redes digitales ayudan a cambiar las relaciones humanas y las formas de comunidad. Las redes digitales, así, hacen parte del "tejido de la sociedad". Y la tecnología comunicacional, reconoce el papa, siendo fruto de la interacción humana, también instaura nuevas dinámicas para esa interacción - una doble hélice en que la sociedad genera nuevas tecnologías para facilitar su interacción, que, por su vez, se ve condicionada y organizada por esas tecnologías, lo que genera nuevos usos sociales creativos o subversivos, demandando nuevas tecnologías, y así por adelante.

En términos más amplios, ocurre un proceso que se encuentra en una interfaz entre lo mediático y lo religioso, en un movimiento creativo y continuo de producción de sentido: o sea, un proceso complejo que apunta para la mediatización de las sociedades contemporáneas. De cierta forma, McLuhan (1964, p. 10, traducción nuestra) ya había antevisto el surgimiento de ese fenómeno al afirmar que "toda tecnología gradualmente crea un ambiente humano totalmente nuevo", ambientes que "non son envoltorios pasivos, pero procesos activos", justamente por ser procesos sociales. Con el avanzo tecnológico y los nuevos procesos sociales correlacionados, se va constatando cada vez más "una aceleración y diversificación

Facultad de Ciencias de la Información - Universidad de La Laguna

Avenida César Manrique, s/n; Campus de Guajara

38071 La Laguna, Tenerife (Islas Canarias - España) 
de los modos por los cuales la sociedad interactúa con la sociedad" (Braga, 2012, p. 35, traducción nuestra).

En ese contexto, entendemos por mediatización un fenómeno social contemporáneo en que los procesos comunicacionales de las sociedades ocurren cada vez más según dinámicas y lógicas mediáticas. En términos generales, la mediatización puede ser entendida como un meta-proceso, segundo Krotz (2007, p. 257, traducción nuestra). Para el autor, meta-procesos son "constructos que describen y explican teóricamente dimensiones y niveles económicos, sociales e culturales específicos de cambio real". Ejemplos históricos de esos meta-procesos son la urbanización, la mercantilización, la individualización, la secularización, la globalización, procesos que duran por siglos y que no están necesariamente confinados a determinada área o cultura, ni se sabe exactamente cuando empiezan o terminan. La mediatización, por lo tanto, puede ser entendida como un meta-proceso comunicacional que nos ayuda a entender la sociedad contemporánea, a partir de la transformación social que se desencadena por medio de procesos socio-mediáticos.

Es ese meta-proceso, entre los demás, continúa Krotz (2007), que molda las condiciones de vida social a longo plazo, tanto en nivel micro (las acciones y las prácticas individuales de producción de sentido), cuanto en nivel mediano (las actividades y estrategias de instituciones y organizaciones sociales religiosas), e aún en nivel macro (la naturaleza de una determinada cultura y sociedad). La mediatización, en ese sentido, se basa en la "modificación de la comunicación como la práctica básica de la forma como las personas construyen el mundo social e cultural” (Krotz, 2007, p.25, traducción nuestra).

En términos religiosos, ocurre, por lo tanto,

El desvío de la mirada del fiel de los templos tradicionales para los nuevos templos mediáticos y digitales, que estimulan, bajo nuevos formatos y protocolos, la experimentación de una práctica religiosa, que encuentra sus raíces en la realidad offline, pero que es ahora resignificada para el ambiente digital [...] O sea, las personas pasan a encontrar una oferta de experiencia religiosa no apenas en las iglesias de piedra, en los sacerdotes de carne e oso e en los rituales palpables, pero también en la religiosidad existente y disponible en los bits e pixels en internet. En esas ofertas de sentido religioso, el fiel, donde quiera que esté, cuando quiera que sea - delante de un aparato electrónico conectado a internet -, desarrolla, así, un nuevo vínculo con la Iglesia y lo sagrado, y un nuevo ambiente de culto. Delante de la pantalla de la 
computadora, entre bits y pixels, el fiel opera la construcción de nuevas formas de alabanza a Dios (Sbardelotto, 2012, p. 26-28, traducción nuestra).

Por eso, es importante percibir "como la religión es constituida hoy en nuevas formas por medio de las culturas digitales" y comprender "la real contribución que 'lo digital' está haciendo a 'lo religioso"' (Hoover, 2012, p. ix, traducción nuestra). En ese sentido, nos proponemos aquí a reflexionar sobre las prácticas sociales de reconexión que ocurren en dispositivos conexiales, a partir del caso "católico".

\section{Reconexiones en dispositivos conexiales: el caso "católico"}

Vemos en las redes sociales digitales una acción social sobre lo "católico" - o sea, constructos simbólicos sobre las creencias y prácticas que la sociedad en su conjunto considera como vinculados a la experiencia y a la tradición de la Iglesia Católica ${ }^{2}$ - mediante una sociotecnicidad específica de la contemporaneidad, que genera nuevas formas de producción de sentido, de experiencia y de discurso religiosos. Nos referimos a las reconexiones en dispositivos conexiales. Profundicemos esos dos conceptos.

El fenómeno al que aquí nos referimos dice respeto a un medio específico, es decir, a la red de redes, internet, y, en su interior, a redes sociales digitales específicas. En su forma más simple, una red es un conjunto de nodos interconectados, que, en nuestro caso, son los internautas en interacción comunicativa. Para Kerckhove (1999), la "esencia de toda red" es precisamente la conectividad. Para el autor, la Internet es "el medio conectado por excelencia, es la tecnología que hace explícita y tangible esta condición natural de la interacción humana" (Kerckhove, 1999, p. 25). Lo conectado, afirma, se convirtió en una alternativa a lo individual y a lo colectivo.

Es importante percibir que "los nodos [de una red] siempre compiten por conexiones, porque los links representan la supervivencia en un mundo conectado" (Barabási, 2003, p.106, subrayado y traducción nuestros). Eso nos lleva a abandonar la idea de las redes como ya dadas, listas, como si bastara analizar los nodos existentes para inferir vínculos, capitales simbólicos etc. La especificidad de las redes socio-digitales no es ser meramente "digitales", ni

${ }^{2}$ Como indica Berger (1979, p. 46), "el núcleo del fenómeno de la religión es un conjunto de experiencias altamente distinctivas", y la religión, de hecho, como el catolicismo, deriva de experiencias religiosas. Pero, para mantener la realidad subjetiva de la experiencia, esta se encarna en tradiciones, "que la median [la experiencia] a aquellos que no la tuvieron personalmente e que la institucionalizan para ellos así como para los que la tuvieron”.

Facultad de Ciencias de la Información - Universidad de La Laguna

Avenida César Manrique, s/n; Campus de Guajara

38071 La Laguna, Tenerife (Islas Canarias - España) 
meramente "sociales" - innumerables otras redes son digitales sin ser sociales, o son sociales sin ser digitales -, sino que su existencia manifiesta una interfaz característica de la contemporaneidad, marcada por vínculos individuales y sociales que se establecen comunicacionalmente mediados por la tecnología digital.

Acreditamos, por eso, que toda red es una acción social de conexión, un trabajo en red (network); o sea, las conexiones no existen "en si mismas", pero son construidas y mantenidas constantemente por la acción social de comunicación mediada por tecnologías digitales. Las redes sociales digitales se establecen no apenas por algo, pero también para hacer algo. En el caso católico, las interacciones sociales que se establecen en Facebook, por ejemplo, utilizan las creencias y las prácticas católicas como eje central de su acción comunicativa, pero también hacen algo sobre esa experiencia y tradición por medio de sus discursos y acciones comunicacionales. $\mathrm{Y}$ lo hacen porque la propia plataforma Facebook insta los usuarios a una producción constante de sentido en su interior, por medio del compartir y del conectar ${ }^{3}$. Así, lo "católico" es el resultado de esa producción pública, conectada y ininterrumpida de sentido social y simbólico sobre las creencias y prácticas católicas, que reconstruye socialmente la experiencia y la tradición del catolicismo.

Vemos un caso de reconexión en el ejemplo que sigue (Fig. 1) ${ }^{4}$.

\footnotetext{
${ }^{3}$ Así dice el "Principio" número 1 de Facebook ("Libertad para compartir y conectarse"): "Las personas deben tener la libertad de compartir las informaciones que desearen, de cualquier manera y en cualquier formato, y tienen el derecho de conectar a cualquier uno - cualquier persona, organización o servicio - desde que ambos estén de acuerdo con la conexión" (traducción nuestra). Disponible en $<$ https://www.facebook.com/principles.php>

${ }^{4}$ Disponible en <http://goo.gl/4sUTf5>.
}

Facultad de Ciencias de la Información - Universidad de La Laguna

Avenida César Manrique, s/n; Campus de Guajara

38071 La Laguna, Tenerife (Islas Canarias - España) 


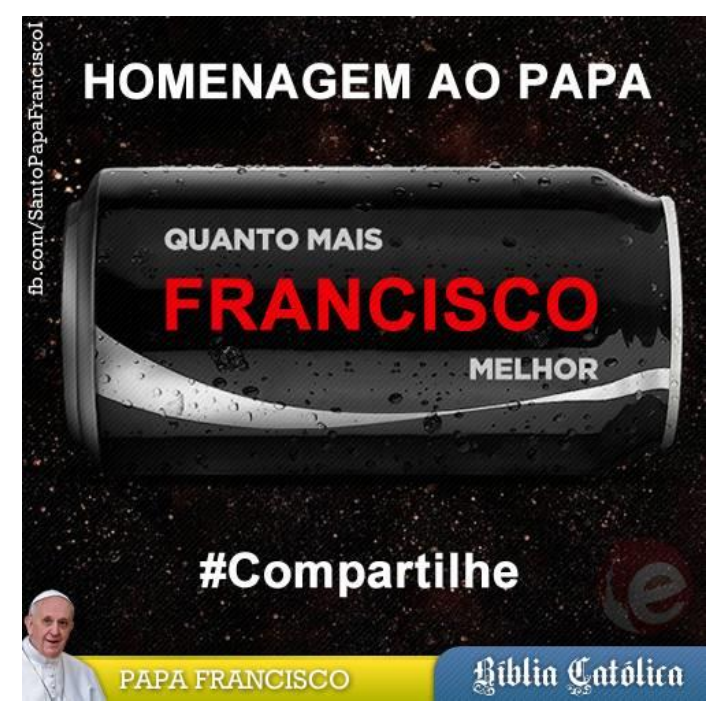

Figura 1 - Post de la página "Papa Francisco I" en Facebook

En ese caso, la página brasileña de Facebook intitulada Papa Francisco $l^{5}$, vinculada a la tradición católica, pero no oficialmente (o sea, no está vinculada a alguna instancia jerárquica de la Iglesia $)^{6}$, publica un "Homenaje al Papa", con una imagen de una lata de gaseosa con la frase "Cuanto más Francisco mejor". La imagen remite a la gaseosa Coca-Cola Zero y a la publicidad que circuló en Brasil que utilizaba el slogan "Quanto mais zero melhor" ("Cuanto más cero mejor"). La imagen, además, propone la invitación en formato de hashtag que dice "\#Comparte".

Vemos, entonces, una acción de reconexión de símbolos de universos completamente distintos, el religioso y el mercadológico-publicitario: los administradores de la página crean un punto de conexión entre esas dos instancias, a principio contrapuestas (el Papa Francisco y Coca-Cola). La reconexión simbólica, por lo tanto, conyuga la "sed" metafórica por la persona del pontífice católico con la sed física de Coca-Cola (cuanto más papa o Coca-Cola, mejor).

\footnotetext{
${ }^{5}$ Disponible en <https://www.facebook.com/SantoPapaFranciscol>.

${ }^{6}$ En el campo "Sobre" de la página, se dice: "Esta página no se trata de un canal oficial. Apenas de un medio de difusión de las noticias relacionadas al Papa Francisco. Administrada por el Portal Ecclesia". Por su vez, el Portal Ecclesia surgió en 2011, a partir del Portal da Capela São José, sin indicación de localización. Se afirma que "hoy somos un equipo de voluntarios y columnistas, que visan, a través de las informaciones, evangelizar a través de los medios de comunicación" - por lo tanto, sin vinculación oficial con la Iglesia (disponible en <http://www.portalecclesia.com/p/institucional.html>).
} 
Es interesante que esa reconexión simbólica es también social, pues es reconocida (más de 770 personas hicieron clic en la opción "Me gusta") o criticada por los demás internautas. Es lo que ocurre en el campo de los comentarios a esa entrada: algunos usuarios felicitan los administradores por crear esa "bella campaña", o dicen que "amaron" la idea; pero otros critican como "ridículo transformar [el papa] en consumo o interpretar como consumo", o dicen que no les gustó ver el nombre del papa en "ese embalaje que es tan visto como símbolo de polución", que es "una falta de respeto", que "el embalaje no merece el contenido". Así, en el campo de los comentarios o por medio de las opciones "Me gusta" y "Compartir", la reconexión social se manifiesta con grande fuerza, reconstruyendo los sentidos simbólicos originales propuestos por los administradores de la página y extendiendo su sentido para otros circuitos comunicacionales.

Por otro lado, las reconexiones simbólicas y sociales permiten aún una reconexión técnica de las funcionalidades de Facebook y de sus contenidos o conexiones. Algunos usuarios, impulsados por la entrada de la página Papa Francisco I, se reapropian del espacio conexial de Facebook transformándolo en un ambiente de oración, como vemos en la figura abajo (Fig. 2).

\section{De Maria Teresa Nunes Correia Concordo. Quanto mais Francisco melhor. Que o Senhor o ajude sempre. \\ 14 de abril às $10: 16 \cdot$ Curtir ' $M 1$

Figura 2 - Comentarios de usuarios en publicación de la página "Papa Francisco I" en Facebook

Como vemos, los usuarios en la página de Facebook, hacen una reconexión técnica, reconstruyendo una funcionalidad de Facebook (el campo de comentarios) como espacio de práctica religiosa pública. "Maria Teresa Nunes Correia" encierra su comentario con la afirmación: "Que el Señor le ayude siempre", en referencia al papa, y "Marcilia Silva" dice: "Demasiado feliz con este papa, igloria a Dios!". De esa forma, Facebook no es apenas un espacio de conexión entre los usuarios, como sugiere la propia plataforma, pero también es 
reconstruido simbólica, social y técnicamente como un ambiente religioso de oración y conexión con el sagrado.

Lo que ocurre en las redes socio-digitales, por lo tanto, son conexiones "nuevas", "ultraconexiones", que van más allá de lo que está dado en términos sociales, técnicos y simbólicos sobre lo religioso. En esas conexiones, se manifiesta la invención social sobre lo "católico" en un proceso específico de circulación comunicacional, marcado por la tecnología digital. Por lo tanto, esas acciones de reconexión permiten partir de algo ya dado y llegar a algo nuevo (invención, in + venire) por medio de "prácticas conectadas" (Miège, 2009), que se suman a las prácticas tradicionales de construcción de lo "católico". Es en la reconexión que los usuarios construyen sentido en red a partir de lo que ya existe social, simbólica o tecnológicamente.

Las interacciones sociales que percibimos en las redes digitales en el ámbito católico, por lo tanto, operan principalmente por medio de reconexiones socio-técnico-simbólicas sobre lo "católico". O sea, son acciones que van más allá de los lazos sociales tradicionales de los individuos e instituciones, conectando de modo nuevo los agentes socio-religiosos; van más de las posibilidades técnicas de las plataformas, conectando de modo nuevo sus funcionalidades con usos religiosos diversos; van más allá de los sentidos "católicos" ya existentes, conectando de modo nuevo los símbolos religiosos entre sí o con otras simbologías no previstas.

Por otro lado, en las interacciones online, es posible ver la manifestación de ciertas matrices, operaciones y modos prácticos compartidos de producción de sentido y de interacción social. Primeramente, es la propia interfaz de Facebook que condiciona ciertos protocolos: por ejemplo, las acciones centrales del usuario son "comentar", "compartir" y "gustar". Otros protocolos van surgiendo en la interacción: algunas páginas de Facebook estipulan y determinan como deben ser (o no ser) los comentarios, creando sanciones y penalidades para los que nos se adecúan a esos protocolos; o, por otro lado, el uso social recurrente de las hashtags ${ }^{7}$, práctica común en Twitter, que después pasó a ser apropiada por la plataforma Facebook etc. O sea, más específicamente, se trata de un dispositivo conexial, un sistema socio-técnico-simbólico heterogéneo de interfaces y protocolos que regulan la conexión digital y organizan la comunicación entre los agentes en rede, sean individuos o instituciones. Los dispositivos conexiales, por lo tanto, establecen redes complejas de interacción social y de circulación comunicacional.

\footnotetext{
${ }^{7}$ Indexadores automáticos de palabras-clave que también sirven como filtro de búsqueda.
} 
Dispositivo, segundo Foucault (1982, p.244), es "un conjunto decididamente heterogéneo que engloba discurso, instituciones, organizaciones [...]. En suma, lo dicho y lo no dicho son los elementos del dispositivo". Los dispositivos disponen el mundo y la sociedad; el mundo y la sociedad disponen los dispositivos; y por medio de ellos la sociedad dispone el mundo. En términos comunicacionales, el dispositivo conexial es algo específico de una dada historicidad (el proceso de mediatización digital), que hace la mediación y organiza las interacciones sociodigitales, o sea, posibilita la propia existencia de la sociedad por medio de trocas más o menos reguladas entre los individuos en red. El dispositivo conexial se estructura a partir de las "configuraciones socio-técnicas" que fornecen "bases reforzadas para la mediatización de la comunicación" (Gresec II apud Miège, 2009, p.187, traducción nuestra).

En el caso analizado anteriormente, el dispositivo conexial es formado por todas las instancias de la interfaz y del protocolo de la plataforma Facebook que posibilitan la conexión digital entre los usuarios: el dispositivo permite la inserción de imágenes y textos por parte del "posteador" a su red de amigos apenas o también de forma pública, siendo puesto a disposición de los sistemas de busca en internet; permite que el lector puede comentar el post, marcar la opción "Me gusta", compartirlo con sus amigos, siendo que todas esas opciones hacen con que el contenido original del "posteador" recircule por nuevas redes de usuarios, ahora del lectorcomentador; permite que cada nuevo comentario puede ser respondido o marcado como "me gusta" por otros usuarios, lo que genera nuevas recirculaciones del contenido y de las mismas conexiones por nuevas redes de usuarios etc. Es ese dispositivo complejo que posibilita las reconexiones.

\section{Consideraciones finales: la emergencia de las "e-rejías"}

En las redes socio-digitales, como vemos, lo "católico" circula y es reconstruido en flujos constantes de circulación comunicacional, mediante una socio-tecnicidad específica de la contemporaneidad como los dispositivos conexiales. En las reconexiones, se desencadena un proceso específico de circulación comunicacional, marcado por ciertas matrices, operaciones y modos prácticos compartidos de producción de sentido y de interacción social, que manifiesta la invención social sobre lo "católico". Eso se suma a las "transformaciones de la sociedad moderna - pluralismo de las concepciones de mundo, privatización y subjetivación del fenómeno religioso - [que] obligan a todos a ser 'herejes', o sea, a realizar una 'libre elección' (en griego: hairesis) entre las religiones y las concepciones de mundo existentes en una dada sociedad" (Martelli, 1995, p.294, traducción nuestra).

Facultad de Ciencias de la Información - Universidad de La Laguna

Avenida César Manrique, s/n; Campus de Guajara

38071 La Laguna, Tenerife (Islas Canarias - España) 
Eso es lo que Berger (1979) llama de "imperativo herético". Si, en la pre-modernidad, la herejía era una posibilidad, en la sociedad contemporánea ella se torna una necesidad, pues es necesario escoger y decidir delante de múltiples posibilidades no solo religiosas en general, pero también "católicas", en que las definiciones y las filiaciones ya no se dan más a priori. "En situaciones pre-modernas, hay un mundo de certeza religiosa, ocasionalmente roto por desvíos heréticos. Al contrario, la situación moderna es un mundo de incerteza religiosa, ocasionalmente evitada por construcciones más o menos precarias de afirmación religiosa" (Berger, 1979, p.28, traducción nuestra). Por lo tanto, de marginal, la "herejía" se torna universal y general.

Ese proceso se complejifica en internet, en que vemos, por medio de las reconexiones en dispositivos conexiales, una religiosidad en experimentación, marcada por la poca fidelidad institucional y doctrinal, por la fluidez de los símbolos en tránsito religioso y por la subjetivación de las creencias: de la herejía tradicional, pasamos a las e-rejías, o sea, "bricolajes de la fe" en el ambiente digital, en que "el propio individuo produce, de manera autónoma, el dispositivo de sentido que le permite orientar su vida y responder a las cuestiones últimas de su existencia", estableciendo "un vínculo entre su solución creyente personal y una tradición creyente instituida a la cual él se reporta de manera libre" (Hervieu-Léger, 2008, p.156-157, traducción nuestra). De esa forma, el fiel "recorta los universos simbólicos - el de su grupo y los ajenos, todos igualmente 'virtuales' - y multiplica los 'colajes', al sabor de una creatividad idiosincrática ('idiosincrética'), radicalmente individual, mismo si se articula en tribus de libre elección" (Sanchis, 1997, p.104).

De esa forma, "hoy, gracias a la 'inteligencia colectiva' fornecida por la red, un simples amador [profano/laico] puede movilizar conocimientos idénticos a los del especialista [sagrado/religioso]" (Flichy, 2010, p.8, traducción nuestra), como en el caso de los administradores de la página Papa Francisco I en Facebook y sus seguidores. Manifiesta-se en esos casos la "reconstrucción del religioso" por parte del internauta común, que actúa sobre los saberes y "desarrolla prácticas refractarias y originales, bricolajes que pueden desembocar en descubiertas" (Flichy, 2010, p.10, traducción nuestra) sobre lo "católico", a partir de las reconexiones. Las actividades del usuario común "no dependen de la constricción de un trabajo o de una institución, sino de una elección. Él es guiado por la curiosidad, por la emoción, por la pasión, por la adhesión a prácticas muchas veces compartidas con los otros" (Flichy, 2010, p.12, traducción nuestra). Así, los procesos productivos de la religión pasan a no ser más realizados ni controlados solamente por la institución eclesial, pero se expanden a todo el corpus social conectado.

Facultad de Ciencias de la Información - Universidad de La Laguna

Avenida César Manrique, s/n; Campus de Guajara

38071 La Laguna, Tenerife (Islas Canarias - España) 
Ese "sensus (in)fidelium" manifestado en internet también posibilita la percepción del desequilibrio entre como el sistema religioso (en términos de institución-Iglesia) es pensado y como es practicado por la sociedad. La turbulencia, la inestabilidad, el desvío provocados por los internautas fomentan la evolución de la experiencia y de la tradición religiosas - en ese caso, rumbo a una apertura sistémica de la Iglesia al pluralismo religioso y cultural del macrosistema social. Se "toda tradición es una memoria colectiva" (Berger, 1979, p. 49), la tradición religiosa es una memoria colectiva de experiencias religiosas, que, en las interacciones sociales digitales, se transforma en memoria conectiva, construyéndose y reconstruyéndose en las reconexiones. De esa forma, por se tratar de una acción sociotécnico-simbólica en el ambiente digital sobre las creencias y las prácticas católicas, las erejías son, al mismo tiempo, relativas (a la experiencia y a la tradición católicas) y absolutas (en su novedad de sentido socio-religioso). Como fruto de lo "católico", las e-rejías son, al mismo tiempo, producto final de la producción de sentido y potencialidad de nuevos sentidos, que pueden contribuir retroactivamente para la reconstrucción social de las creencias y de las prácticas sobre lo "católico" que las construyeron (cf. Morin, 2008).

Em suma, las redes socio-digitales instauran una nueva modalidad de comunicación, activando un "dispositivo de publicización del discurso laico" (Blanchard apud Miège, 2009, p.169, traducción nuestra). El fiel, por lo tanto, no es apenas co-constructor de su fe personal, pero también realiza un "trabajo creativo" sobre la propia religión, tensionando la "interfaz eclesial". Así, en el caso católico, ocurre un desvío de la autoridad eclesial y una correlata autonomización del fiel en las prácticas religiosas. En ese sentido, "las tecnologías digitales son profundamente marcadas por los comportamientos de autonomía individual y de 'conectividad' [mise en connexion]" (Flichy, 2010, p.15, traducción nuestra), contribuyendo para el desarrollo de nuevas prácticas sociales y religiosas.

Así, mediante reconexiones en dispositivos conexiales, la sociedad como un todo - como un masaje - trabaja, satura, modela, reconstruye y transforma todas las relaciones de los sentidos religiosos (cf. Mcluhan in Mcluhan \& Zingrone, 1998).

\section{Bibliografía}

Benedicto XVI (2012). Redes sociales: Portales de verdad y de fe; nuevos espacios para la evangelización. Mensaje para la 47ํㅡㄹ Jornada Mundial de las Comunicaciones Sociales.

Vaticano: Libreria Editrice Vaticana. Disponible en <http://migre.me/egwil>. 
Berger, P. L. (1979). Heretical Imperative: Contemporary Possibilities of Religious Affirmation. New York: Doubleday.

Braga, J. L. (2012). Circuitos versus campos sociais. In: Mattos, M. A.; Junior, J. J.; Jacks, N. (orgs.). Mediações e midiatização (pp. 31-52). Salvador: EDUFBA.

Fausto Neto, A. (2010). A circulação além das bordas. In: Fausto Neto, A.; Valdettaro, S. Mediatización, sociedad y sentido: Diálogos entre Brasil y Argentina (pp. 2-17). Rosario: UNR. Disponible en $<$ http://migre.me/d9NaP>.

Flichy, P. (2010). Le sacre de l'amateur: Sociologie des passions ordinaires à l'ère numérique. Paris. Éditions du Seuil.

Foucault, M. (1982). Microfísica do poder. $3^{\text {a }}$ ed. Rio de Janeiro: Graal.

Hervieu-Léger, D. (2008). O peregrino e o convertido: a religião em movimento. Petrópolis: Vozes.

Hoover, S. M. (2012). Foreword: Practice, Autonomy, and Authority in the Digitally Religious and Digitally Spiritual. In: Cheong, P. H.; Nielsen-Fischer, P.; Gelfgren, S.; Ess, C. (eds.). Digital Religion, Social Media and Culture: Perspectives, Practices and Futures (pp. vii-xii). New York: Peter Lang Publishing.

Hoover, S. M.; Echchaibi, N. (2012). The "Third Spaces" of Digital Religion. Boulder: The Center for Media, Religion, and Culture. Disponible en <http://migre.me/8xlqG $>$.

Kerckhove, D. D. (1999). Inteligencias en conexión: Hacia una sociedad de la web. Barcelona: Gedisa.

Krotz, F. (2007). The Meta-Process of 'Mediatization' as a Conceptual Frame. Global Media and Communication, 3, 256-260. Disponível em <http://migre.me/4Cvan >.

Martelli, S. (1995). A religião na sociedade pós-moderna: Entre secularização e dessecularização. São Paulo: Paulinas.

McLuhan, M. (1964). Os meios de comunicação como extensões do homem. São Paulo: Cultrix.

McLuhan, E.; Zingrone, F. (eds.) (1998). McLuhan: Escritos esenciales. Barcelona: Paidós.

Miège, B. (2009). A sociedade tecida pela comunicação: Técnicas da informação e da comunicação entre inovação e enraizamento social. São Paulo: Paulus. 
Morin. E. (2008). O método 1: A natureza da natureza. $2^{\mathrm{a}}$ ed. Porto Alegre: Sulina.

Sanchis, P. (1997). O campo religioso contemporâneo no Brasil. In: Oro, A. P.; Steil, C. A. (eds.). Globalização e Religião (pp. 103-116). Petrópolis, Vozes.

Sbardelotto, M. (2012). E o Verbo se fez bit: A comunicação e a experiência religiosas na internet. Aparecida: Santuário.

Sínodo de los Obispos (2012). La nueva evangelización para la transmisión de la fe cristiana. Instrumentum laboris de la XIII Asamblea General Ordinaria. Ciudad del Vaticano: Libreria Editrice Vaticana. Disponível em <http://migre.me/egvXd>.

\section{Forma de citar este artículo en bibliografías}

SBARDELOTTO, M. (2013). "Reconexiones en dispositivos conexiales: La reconstrucción de lo religioso en redes socio-digitales" se encuentra publicado en Revista PANGEA (ISSN: 2172 - 3168); Año 2013, № 2, en páginas 103 a 117. Red Académica Iberoamericana de Comunicación. Recuperado el _ de _ de 2 _ de: http://www.revistapangea.org 